\title{
Cefazolin Sodium
}

National Cancer Institute

\section{Source}

National Cancer Institute. Cefazolin Sodium. NCI Thesaurus. Code C47968.

The sodium salt of cefazolin, a beta-lactam antibiotic and first-generation cephalosporin with bactericidal activity. Cefazolin binds to and inactivates penicillin-binding proteins (PBP) located on the inner membrane of the bacterial cell wall. Inactivation of PBPs interferes with the cross-linkage of peptidoglycan chains necessary for bacterial cell wall strength and rigidity, which results in the weakening of the bacterial cell wall and cell lysis. 